\title{
Differentially expressed proteins in platelets derived from patients with hypertension
}

\author{
Yobana Armenta-Medina $\mathbb{1}^{1} \cdot$ Ivette Martínez-Vieyra ${ }^{1} \cdot$ Oscar Medina-Contreras $\mathbb{D}^{2} \cdot$ Claudia G. Benitez-Cardoza $^{3}$. \\ Albertana Jiménez-Pineda $\mathbb{1}^{3} \cdot$ César A. Reyes-López $\mathbb{1}^{4} \cdot$ Doris Cerecedo $\mathbb{1}^{1}$
}

Received: 19 February 2021 / Revised: 7 May 2021 / Accepted: 20 May 2021 / Published online: 3 July 2021

(c) The Author(s), under exclusive licence to Springer Nature Limited 2021

\begin{abstract}
Hypertension (HTN) causes end-organ damage and is a major cause of morbidity and mortality globally. Recent studies suggested blood cells participate in the maintenance of HTN. Platelets—anucleated cell fragments derived from megakaryocytes-exert diverse functions, including their well-characterized role in the formation of hemostatic clots. However, platelets from patients with HTN exhibit altered membrane lipid and protein compositions that impact platelet function and lead to formation of aggregates and vascular obstructions. Here, for the first time, we have identified, by proteomic analyses, the most relevant 11 proteins that show the greatest difference in their expression in platelets derived from patients with HTN, in comparison with those from normotensive individuals. These proteins are involved in cytoskeletal organization and the coagulation cascade that contributes to platelet activation, release of granule contents, and aggregation, which culminate in thrombus formation. These results have important implications in our understanding of the molecular mechanisms associated with the development of HTN, and in consequence, the development of new strategies to counteract the cardiovascular disorders associated with constitutive activation of platelets in HTN.
\end{abstract}

\section{Introduction}

Hypertension (HTN) is a common, multifactorial disease and the major risk factor for heart attacks, stroke, and kidney failure. Furthermore, HTN has been implicated in higher susceptibility and adverse outcomes of patients infected with the current pandemic caused by SARS-CoV2, thereby

Supplementary information The online version contains supplementary material available at https://doi.org/10.1038/s41371021-00555-y.

$\triangle$ Doris Cerecedo

dcereced@ipn.mx

1 Laboratorio de Hematobiología, ENMyH, Instituto Politécnico Nacional, Mexico City, Mexico

2 Unidad de Investigación Epidemiológica en Endocrinología y Nutrición, Hospital Infantil de México Federico Gómez, Mexico City, Mexico

3 Laboratorio de Investigación Bioquímica, ENMyH, Instituto Politécnico Nacional, Mexico City, Mexico

4 Laboratorio de Bioquímica Estructural, ENMyH, Instituto Politécnico Nacional, Mexico City, Mexico rejuvenating our interest in better understanding this condition [1]. Mechanisms such as overactivation of the renin-angiotensin-aldosterone system and sympathetic nervous system, hypertrophy, endothelial dysfunction, and oxidative stress have been related the pathogenesis of HTN [2].

HTN is characterized by spontaneous platelet aggregation due to increased platelet activation. Hemodynamic and vascular factors generated by the higher sheer forces in patients with HTN, as well as atherosclerosis, contribute to platelet activation [3]. In addition, the release of higher concentrations of catecholamines and increased sensitivity of platelets to these patients with HTN also plays key roles in platelet activation [4].

Activated platelets undergo a dramatic change in shape from disc shaped to a spherical morphology due to dynamic reorganization of cytoskeletal components, including assembly and disassembly of structural proteins. The percentage of platelets exhibiting active actin polymerization increases from $\sim 35-40$ to $70 \%$ during the activation process. In turn, activated platelets form filopodia and lamellipodia adhere to each other and release their granule contents [5].

Platelets from patients with HTN are constituently activated and exhibit morphological, biophysical, biochemical, 
and functional modifications [6]. Recently, we reported that platelets from patients with HTN possess numerous long filopodia, exhibit deregulated expression of cytoskeletal proteins, and overexpress the epithelial sodium channel. These changes were associated with altered phospholipid and cholesterol contents and changes in the microviscosity of the plasma membrane [7]. Atomic force microscopy and liquid chromatography-tandem mass spectrometry (LC-MS/MS) confirmed the changes in fluidity and altered lipid contents in platelets from patients with HTN compared to platelets from normotensive individuals (NTI). In addition, transmission electron microscopy revealed changes in the inner membranous structure of platelets from patients with HTN [8].

Proteomic analysis provides an important tool to identify proteins; $\sim 80 \%$ of the total protein content of platelets has been characterized [9]. The aim of the present study was to identify differentially expressed proteins in platelets derived from patients with HTN compared to platelets from NTI. Using 2D electrophoresis, we resolved whole platelet proteins from nine patients with HTN and nine NTI. The 11 most importantly differentially expressed electrophoretic entities, between the two groups, were identified using a LC-MS/MS approach and an interactome of the identified proteins was built using STRING. This information may help to identify specific therapeutic target proteins to ameliorate or avoid thrombotic events, or identify biomarkers of disease progression.

\section{Materials and methods}

\section{Reagents}

SDS, acrylamide, bis-acrylamide, urea, thiourea, CHAPS, TEMED, DTT, Tris, TCA, acetic acid, ethanol, Coomassie brilliant blue G 250, aluminum sulfate, and orthophosphoric acid were purchased from Sigma Chemical Co. (St. Louis, MO, USA). Immobilized $\mathrm{pH}$ gradient strips (pH 3-10) and the $2 \mathrm{D}$ electrophoresis reagents and electrophoresis instruments were obtained from Bio-Rad Laboratories Inc. (Hercules, CA, USA).

\section{Subjects}

Human peripheral blood samples were collected from patients diagnosed with essential systemic arterial HTN $(n=9)$ and NTI $(n=9)$ after obtaining signed informed consent. This project was approved by the ethics committee of the ENMyH Instituto Politécnico Nacional (No. 001/2019). Table 1 summarizes the anthropometric characteristics of the two groups. Mean platelet volume (MPV) and platelet count were measured from whole
Table 1 General characteristics of hypertensive patients and controls.

\begin{tabular}{llll}
\hline Parameter & Hypertensive & Controls & $p$ value \\
\hline Age \pm SD (years) & $55.83 \pm 9.26$ & $49 \pm 12.56$ & 0.2439 \\
Sex (\%male/\%female) & $38 / 62$ & $40 / 60$ & - \\
SBP (mmHg) & $149 \pm 23$ & $117 \pm 5$ & 0.0019 \\
DBP (mmHg) & $89 \pm 8$ & $79 \pm 4$ & 0.0001 \\
BMI & $28 \pm 5$ & $27 \pm 4$ & $0.0898 \mathrm{~ns}$ \\
Glucose (mg/dl) & $105 \pm 11.5$ & $89 \pm 5$ & 0.0001 \\
Total cholesterol (mg/dl) & $185.8 \pm 10$ & $191 \pm 13$ & $0.1815 \mathrm{~ns}$ \\
Triglycerides (mg/dl) & $220.4 \pm 52.5$ & $140 \pm 38$ & 0.0001 \\
MPV (fl) & $9.1 \pm 0.84$ & $8.7 \pm 0.6$ & 0.403 \\
Platelet count $\left(\times 10^{9}\right)$ & $161 \pm 21.9$ & $235 \pm 30.3$ & 0.0007 \\
Hypertension grades $(\%)$ & & & \\
Controlled & 44.4 & - & \\
1 & 44.4 & - & \\
2 & 11.2 & - & \\
\hline
\end{tabular}

Values are the mean \pm SD of ten patients and controls in each group. $B M I$ body mass index, $S B P$ systolic blood pressure, $D B P$ diastolic blood pressure, $M P V$ mean platelet volume, $n s$ non significant.

blood samples in an automatic blood counter (Beckman Coulter) within $30 \mathrm{~min}$ after venipuncture.

HTN grades were defined according to 2013 ESC/ESH Guidelines for the management of arterial HTN. Controlled BP was defined as systolic blood pressure (SBP) $<140 \mathrm{mmHg}$ and/or diastolic blood pressure (DBP) < $90 \mathrm{mmHg}$; grade 1 was defined as SBP 140-159 $\mathrm{mmHg}$ and/or DBP 90-99 $\mathrm{mmHg}$; grade 2 was defined as SBP $160-179 \mathrm{mmHg}$ and/or DBP $100-109 \mathrm{mmHg}$; and grade 3 was defined as $\mathrm{SBP} \geq 180 \mathrm{mmHg}$ and/or $\mathrm{DBP} \geq$ $110 \mathrm{mmHg}$.

All subjects maintained their usual diets; the patients with HTN maintained their treatment, which included enalapril, losartan, telmisartan, or amlodipine; no platelet aggregation inhibitors were prescribed. The NTI group had not received any drug treatment during the previous 10 days. Exclusion criteria included pregnancy, smoking, valvular heart disease, type 2 diabetes mellitus, and dyslipidemia. For the 2D gel analysis the patients were grouped regardless the treatment they were receiving.

\section{Platelet sample preparation}

Platelet isolation, protein extraction, and protein preparation for analysis were performed as previously reported by García et al. [10].

LC-MS/MS approach analyzes many proteins in a single experiment, this methodology is not suitable to assess large number of individuals; the washed platelet suspensions were pooled in groups of three to produce three samples for patients with HTN and three samples for NTI. Each pooled 
sample was assessed on three independent gels; therefore, a total of nine gels for patients with HTN and nine gels for NTI were obtained and analyzed.

One thousand two hundred and fifty micrograms of total protein extracts were solubilized in a rehydration buffer (8 M urea, $2 \mathrm{M}$ thiourea, CHAPS 2\%, $50 \mathrm{mM}$ DTT) supplemented with $1 \%$ Bio-Lyte $^{\mathrm{TM}}$ ampholyte (Bio-Rad)), and were loaded onto $17 \mathrm{~cm}$ immobilized $\mathrm{pH}$ gradient IPG strips (pH 3-10). The isoelectric focusing (IEF) was performed using a Protean IEF cell system (Bio-Rad). The IPG strips were actively rehydrated at $50 \mathrm{~V}$ for $14 \mathrm{~h}$, then voltage ramping steps were applied $(100 \mathrm{~V}$ for $2 \mathrm{~h}, 250 \mathrm{~V}$ for $30 \mathrm{~min}, 500 \mathrm{~V}$ for $30 \mathrm{~min}, 10,000 \mathrm{~V}$ for $2 \mathrm{~h}$, for a total $43,000 \mathrm{Vh}$ ) at a maximum current of $50 \mu \mathrm{A}$ per strip. After IEF, the strips were incubated in equilibration buffer (6 M urea, 2\% SDS, $0.375 \mathrm{mM}$ Tris- $\mathrm{HCl}, 20 \%$ glycerol) containing $2 \%$ DTT for $15 \mathrm{~min}$ with gentle agitation, and then placed in equilibration buffer containing $135 \mathrm{mM}$ iodoacetamide for $20 \mathrm{~min}$ in the dark.

Second-dimension protein separation was carried out on $12 \%$ SDS-polyacrylamide gel electrophoresis (SDS-PAGE) gels $(19 \times 21 \mathrm{~cm})$ using a Protean II XL system (Bio-Rad). The equilibrated strips were placed horizontally onto gels (two batches of three simultaneous gels) and the separation was carried out at $15 \mathrm{~mA} / \mathrm{gel}$ at $15^{\circ} \mathrm{C}$.

Following electrophoresis, the gels were fixed in $15 \%$ acetic acid (v/v) followed by $1 \%$ ethanol (v/v) and stained overnight with silver Coomassie $(0.02 \% \mathrm{w} / \mathrm{v}$ Coomassie brilliant blue G $250,5 \% \mathrm{w} / \mathrm{v}$ aluminum sulfate, $10 \% \mathrm{v} / \mathrm{v}$ ethanol [96\%], 2\% v/v orthophosphoric acid [85\%]), prepared as described by with gentle agitation at $4{ }^{\circ} \mathrm{C}$. The gels were destained with $10 \% \mathrm{v} / \mathrm{v}$ ethanol $(96 \%)$ and $2 \% \mathrm{v} / \mathrm{v}$ orthophosphoric acid (85\%), washed with deionized water, and stored for $48 \mathrm{~h}$ at $4{ }^{\circ} \mathrm{C}$.

The gels were scanned using a ChemiDoc System operated using ChemiDoc MP Imaging System software (Bio-Rad). The scanned images were analyzed, and the protein spots were quantified using PDQuest 2D analysis software (Bio-Rad). The workflow included manual alignment of the digitized gel images, creation of the master gel, spot detection, determination of spot volume (product of the OD value per area $\left[\mathrm{OD} \times \mathrm{mm}^{2}\right]$ ), and quantification of the number of spots. All the here-identified spots were at least expressed in 81 and $55 \%$ of the 2D gels from HTA and NTI, respectively.

\section{Nanoflow LC-MS/MS}

Protein spots with the largest differential expression were mechanically excised from the gels and the proteins were digested with trypsin. Protein identification was performed by Alicia Chagolla at the Centro de Investigación y de Estudios Avanzados of the Instituto Politécnico Nacional,
Irapuato, México. All experiments were performed on a nanoAcquity nanoflow LC system (Waters Corporation) coupled to a linear ion trap LTQ Velos mass spectrometer (Thermo Fisher Scientific, Bremen, Germany) equipped with a nanoelectrospray ion source. Solvent A was $0.1 \%$ formic acid and solvent B was $100 \%$ acetonitrile in $0.1 \%$ formic acid. Protein digestate samples $(3 \mu \mathrm{l})$ were bound to a Symmetry ${ }^{\oplus} \mathrm{C} 18$ pre-column $(5 \mu \mathrm{m}, 180 \mu \mathrm{m} \times 20 \mathrm{~mm}$, Waters Corporation). The flow was subsequently switched to a $10-\mathrm{cm}$ capillary UPLC column (100 $\mu \mathrm{m}$ ID BEH-C18, 1.7- $\mu \mathrm{m}$ particle size). Column temperature was controlled at $35^{\circ} \mathrm{C}$. Peptides were separated using a 60-min gradient at a flow rate of $400 \mathrm{nl} / \mathrm{min}$. The gradient was programmed as follows: $3-50 \%$ solvent B (over $30 \mathrm{~min}$ ), 50-85\% B (over $2 \mathrm{~min}$ ), $85 \% \mathrm{~B}$ (over $4 \mathrm{~min}$ ), and $3 \% \mathrm{~B}$ (over $22 \mathrm{~min}$ ). The peptides were eluted into the mass spectrometer nanoelectrospray ion source through a standard coated silica tip (New Objective, Woburn, MA, USA). The mass spectrometer was operated in data-dependent acquisition mode to automatically alternate between full scans (400-1600 m/z) and subsequent top five MS/MS scans in the linear ion trap. CID was performed using helium as the collision gas at a normalized collision energy of $35 \%$ and $10 \mathrm{~ms}$ activation time. Data acquisition was controlled by Xcalibur 2.3 software (Thermo Fisher Scientific).

Tandem mass spectra were extracted to Proteome Discoverer software version 1.4 (Thermo Fisher Scientific Inc., Waltham MA, USA) and subjected to Sequest HT searches against the Human_Uniprot database containing 72,830 sequences and Human_Platelet database containing 977 sequences. Searches were executed using the following parameters: 2 Da parent MS ion window, 1 Da MS/MS ion window, and two missed cleavages allowed. The iodoacetamide derivative of cysteine (carbamidomethylcysteine) was specified as a fixed modification and oxidation of methionine, as a variable modification.

\section{Construction of the interactome}

Association networks for the identified proteins were classified and constructed using the STRING database (Search Toll for Retrieval of Interacting Genes/Proteins, https://string-db.org/). The UniProt ID was entered for each identified protein, and Homo sapiens was selected as the organism.

\section{Statistics}

To ensure comparable participation between HTN patients and NTI, minimize the variability of the evaluation, and provide unbiased evaluation of the proteome analysis, simple randomization was used. We assigned a progressive number to patients and controls when they were considered 
as candidates to be included in the experimental groups; their samples were pooled in groups of three (from 1 to 3 ; from 4 to 6 ; and from 7 to 9 ).

The assessment of normality of data was performed with the Shapiro-Wilk test and the $p$ value of the test corresponded to 0.881 from patients with HTN and 0.946 for NTI, which indicates normal distribution of data for both kinds of samples.

Values are reported as mean \pm SEM and were compared using unpaired $t$-tests. The Pearson correlation $(r)$ was used to evaluate the correlation between theoretical and experimental $\mathrm{pI}$ and MW values.

Statistical and quantitative analyses of the spot changes on the images were performed using PDQuest software (Bio-Rad).

\section{Results}

In the nine patients with HTN, the average age was $55.8 \pm$ 9.26 years, while in the nine NTI the average age was $49 \pm$ 11.56. Male accounted for $38 \%$ of the HTN patients, while $62 \%$ of female were included for this same condition. A similar percentage of NTI males $(40 \%)$ and females $(60 \%)$ was also included. The average SBP $(\mathrm{mmHg})$ for patients with HTN was significantly higher than the corresponding NTI (149.30 \pm 23 vs $117 \pm 5 ; p=0.0019)$, while the average of DBP $(\mathrm{mmHg})$ for patients with HTN was also significantly higher compared to NTI $(89 \pm 8$ vs $79 \pm 4 ; p=$ $0.001)$. The body mass index and total cholesterol values were similar for both populations (HTN patients and NTI), while glucose and triglycerides levels were significantly higher for HTN patients than for NTI $(105 \pm 11.5$ vs $89 \pm 5$; $p=0.0001$ and $220.4 \pm 52.5$ vs $140 \pm 38 ; p=0.0001$, respectively). The MVP values were also similar for HTN patients and for NTI $(9.1 \pm 0.84$ vs $8.7 \pm 0.6)$ in contrast to the significantly lower platelet counts for HTN patients compared to NTI $(161 \pm 21.9$ vs $235 \pm 30.3 ; p=0.0007)$. The distribution of HTN grade was $44.4 \%, 44.4$, and $11.2 \%$ for controlled, grade 1 and grade 2 , respectively (Table 1).

All HTN patients included in this study were prescribed with antihypertensive drugs and in Table 2, we summarize the pharmacotherapy they were receiving at the moment of the inclusion in the study. Four patients were treated with ACE inhibitor in combination with calcium antagonists of dihydropyridine, one patient received ACE inhibitor only, four patients received only the ATI-receptor antagonist, and four patients received ATI-receptor antagonist in combination with a diuretic.

The protein spots on the HTN gels were more intense and clear than the spots on the NTI gels; however, the spots were well-defined for both groups (Fig. 1). The spots were
Table 2 Antihypertensive treatment of hypertensive patients included in the study.

\begin{tabular}{ll}
\hline Treatment prescribed & Number of patients \\
\hline ACE inhibitors & 1 \\
ATI-receptor antagonists & 4 \\
$\begin{array}{l}\text { ACE inhibitors + calcium antagonists of } \\
\text { dihydropyridine }\end{array}$ & 1 \\
$\begin{array}{l}\text { ATI-receptor antagonists + thiazide-like } \\
\text { diuretics }\end{array}$ & 4 \\
\hline
\end{tabular}

$A C E$ angiotensin-converting enzyme inhibitor, $A T 1$ angiotensin II receptor subtype 1 .

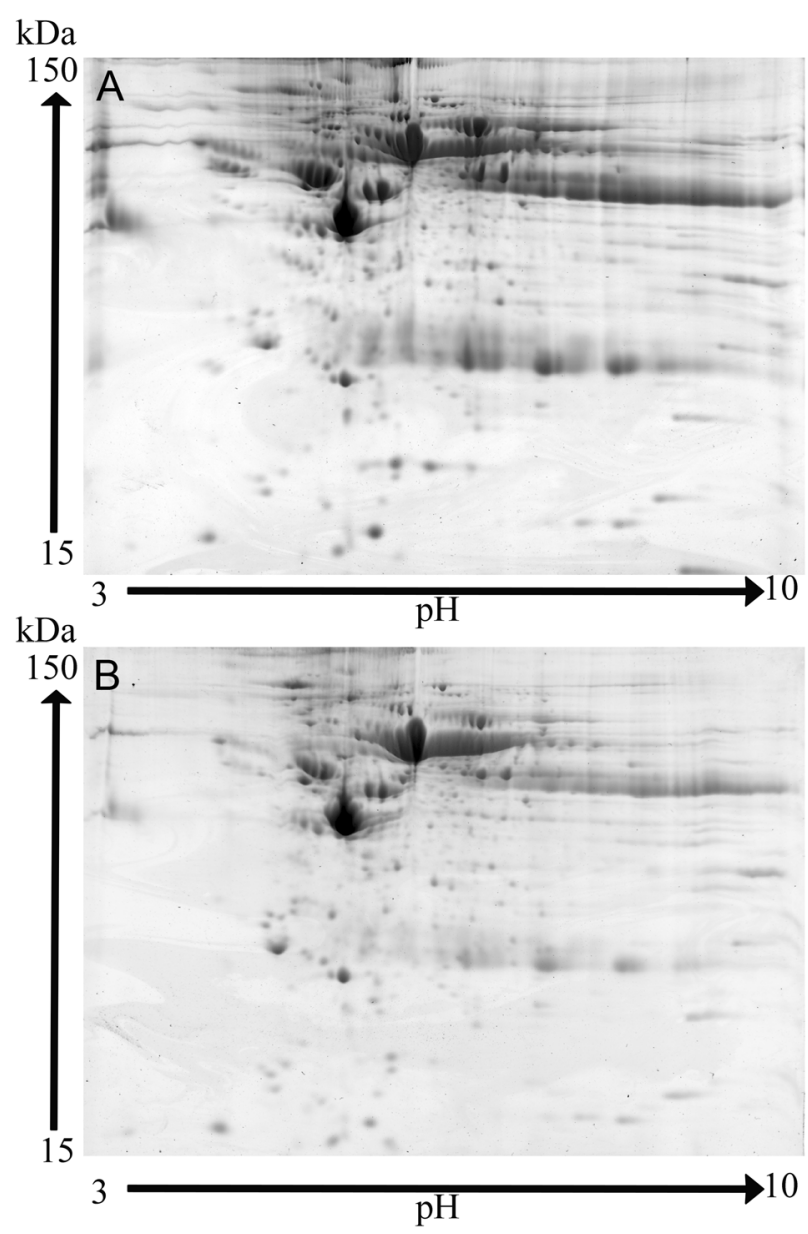

Fig. 1 2D SDS-PAGE of platelets from HTN patients and NTI. Representative 2D silver Coomassie-stained SDS-PAGE gels of A platelets from patients with hypertension (HTN) and B platelets from normotensive individuals (NTI). Proteins were separated over the $\mathrm{pH}$ range of 3-10.

mainly detected at $\mathrm{pH}$ values from 4 to 7 and ranged in size from 15 to $150 \mathrm{kDa}$ (Fig. 1).

Spot analysis identified $163 \pm 9$ spots in the NTI gels and $273 \pm 9$ spots in the HTN gels. The experimental molecular weight, pI, and optical density (arbitrary units) of 159 spots are summarized in Supplementary Table 1; proteins from 


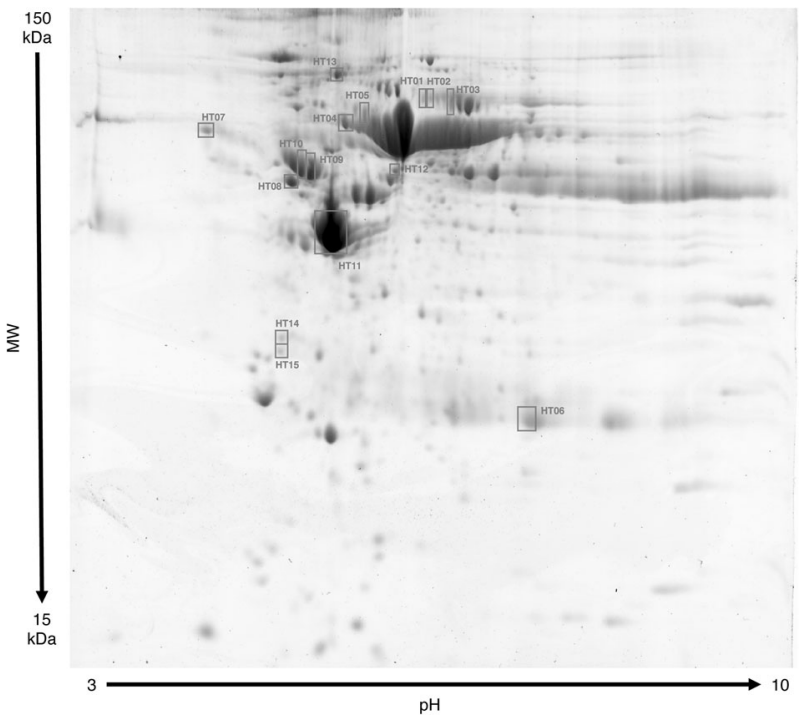

Fig. 2 Positions of significantly differed spots on a 2D gel. Representative 2D SDS-PAGE gel showing significantly differentially expressed protein spots in platelets from patients with HTN; the positions of the 15 spots selected for analysis by LC-MS/MS are indicated.
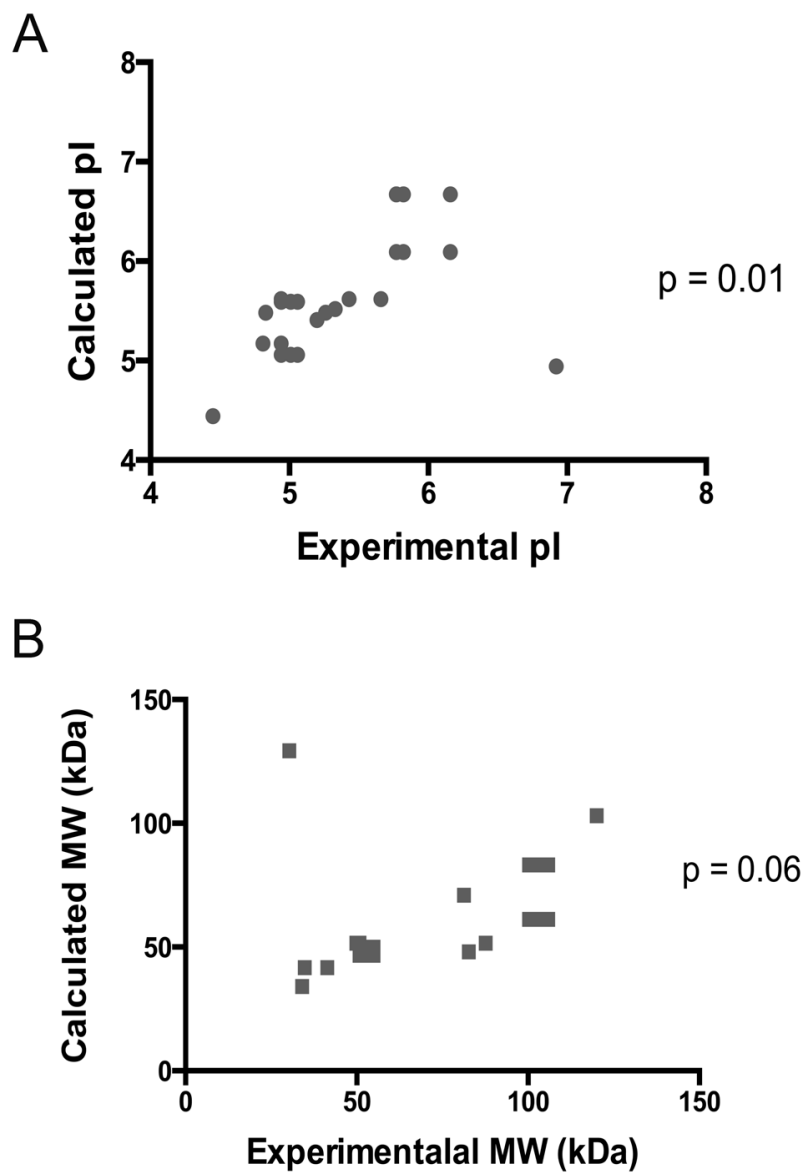

Fig. 3 pI and Mw correlation plots. Correlations between the calculated (theoretical) and experimental A pI values and B MW values for the 15 spots analyzed by LC-MS/MS. the HTN samples are represented in red, while proteins from the NTI samples appear in blue (Table 1S). We detected 73 differentially expressed spots between the HTN gels and the NTI gels with a fold change (fc) $\geq 2(p<0.1)$. Among them, the 15 electrophoretic entities corresponding to the highest fcs and the most well-defined isolated spots or groups of spots (Fig. 2) between the two experimental groups were selected to be analyzed by LC-MS/MS. The experimental $\mathrm{pI}$ and $\mathrm{MW}$ values were estimated from the 2D gels using PDQuest software. The Pearson correlations between the theoretical and experimental $\mathrm{pI}$ and $\mathrm{MW}$ values of the 15 identified proteins were $0.520(p=0.01)$ and 0.395 ( $p=0.06$ ), respectively (Fig. 3A, B). Eleven different proteins corresponding to the 15 selected electrophoretic entities were identified (Table 3 ).

The median CV for pooled sample in LC-MS/MS corresponded to 0.39 and 0.43 for HTN patients and NTI, respectively.

Figure 4 shows the expression profiles of spots in selected areas of representative gels from each group of 15 spots identified by LC-MS/MS, below of each pair of spots the fc is indicated. Five of the spots were overexpressed (positive values) in HTN samples in relation to NTI, while ten were downexpressed (negative values).

From the 11 different proteins, we created an interactome using the STRING database (Fig. 5). Likely functional relationships exhibit scores larger than 0.400 (medium confidence; Fig. 5). A low score was observed for the interaction between actin and calreticulin (0.476), a high score was obtained for the interaction between fibrinogen gamma chain and coagulation factor XIII A chain (0.969), and the highest score was observed for zyxin and alphaactinin (0.980). The functional relationships identified were classified as known interactions (from experimentally determined databases) or predicted interactions (neighbor genes or coexpressed genes).

\section{Discussion}

Blood cells from patients suffering from arterial HTN exhibit distinct biochemical and morphological differences, which strongly suggest dysregulation of several proteins and may contribute to the pathophysiology of the disease.

Our proteomic analysis based on LC-MS/MS focused on the changes in proteins associated with either platelet cytoskeleton, cytoskeleton-interacting proteins, or proteins related with the coagulation system; our results provide specific evidence of physiological changes in the platelets of patients with HTN compared to platelets from NTI.

Platelet size is an indicator of platelet activation in patients with HTN. These patients exhibit lower platelet counts, which could inversely correlate with higher MPV 


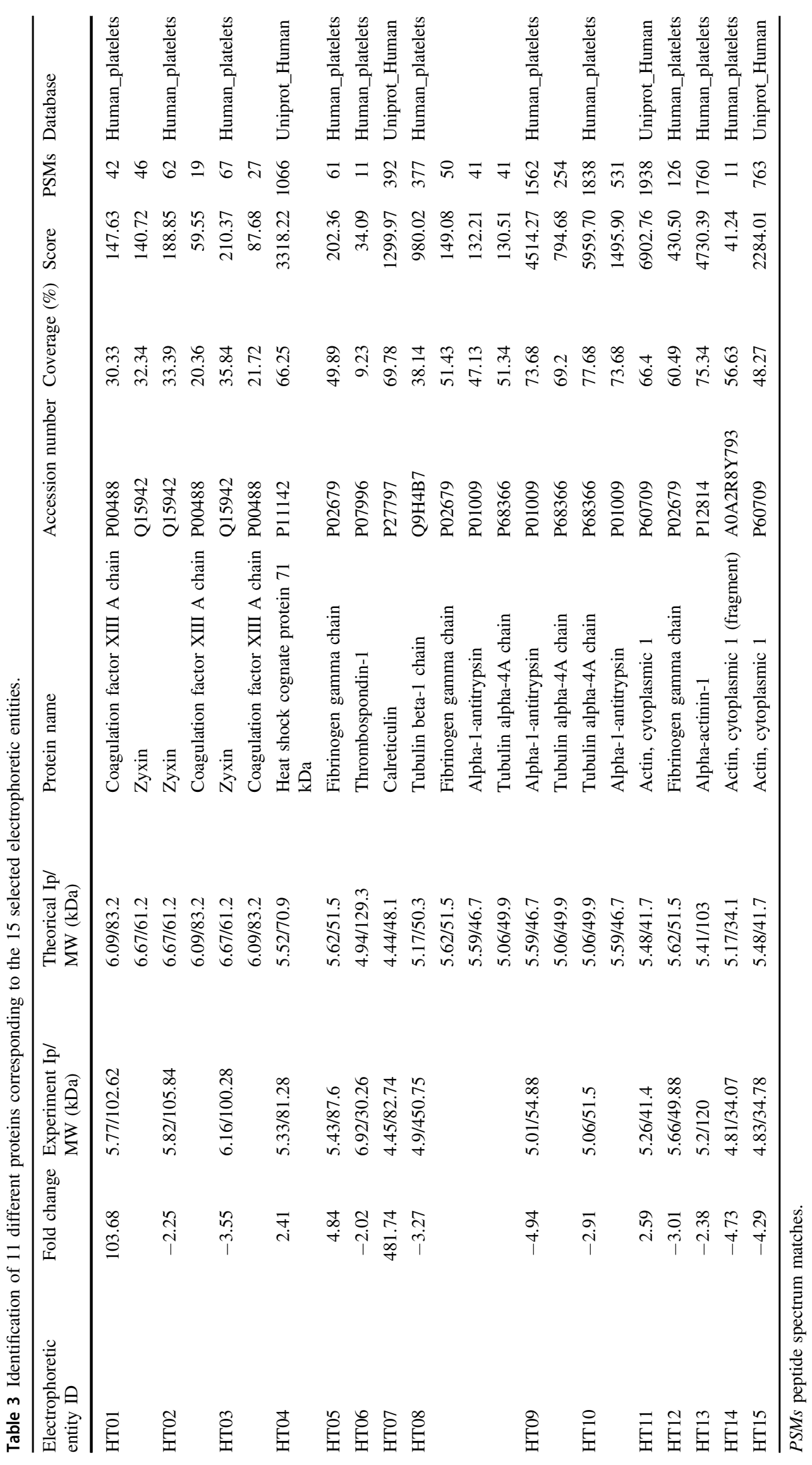



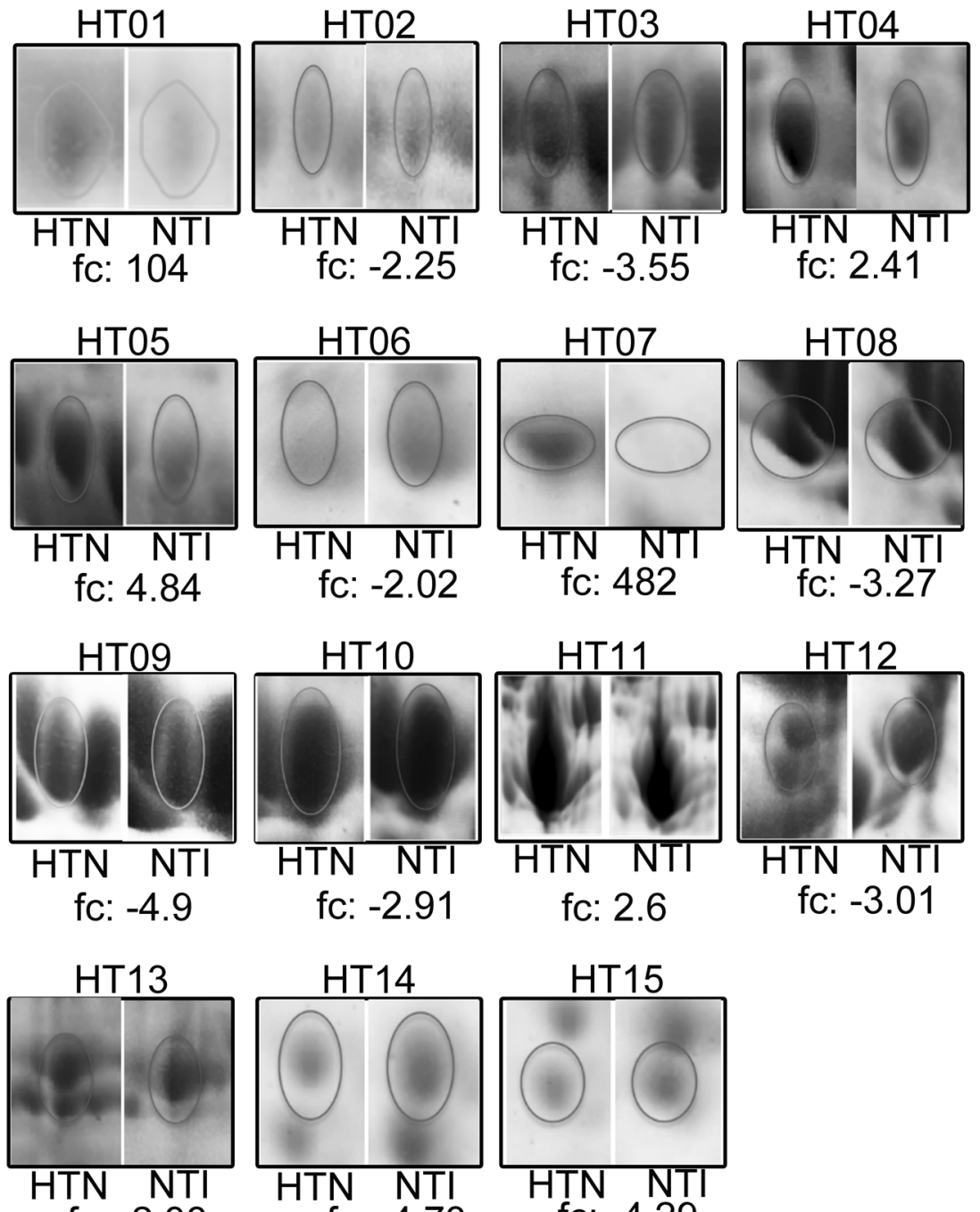

fc: 2.6

fc: -3.01

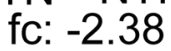

fC: -4.73

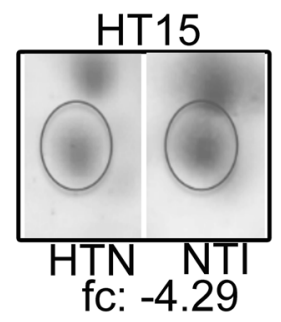

Fig. 4 Magnification of fifteen detected spots of platelets from HTN patients and NTI. Magnification of the differentially expressed positions/ spots (of the 15 differentially expressed positions/spots, fold change $\geq 2$ in platelets from patients with HTN compared to NTI. fc fold change value.

when compared to NTI [11]. In the present study, the MPV values determined for patients with HTN were similar to the MPV observed for NTI as has been reported [12], although HTN patients had significantly lower platelet counts.

We identified 15 differentially expressed spots, 12 have been reported before in platelets from HTN patients or in vitro activated platelets $[10,13]$. Interestingly, we report a previously unidentified underexpression of alpha-1antitrypsin and tubulin alpha- $4 \mathrm{~A}$ chain, as well as the overexpression of calreticulin in HTN patients.

Moderate correlations were observed between the experimental and theoretical isoelectric points (0.520) and molecular weights $(0.395)$ of these 11 proteins. The strength of these correlations might be affected by the narrow $\mathrm{pH}$ range (4-7) and wide range of molecular weights (15-150 $\mathrm{kDa})$. In addition, numerous posttranslational modifications may alter the $\mathrm{pI}$ and/or the MW of proteins and thus shift the position of protein spots in 2D gels [14].

The proteins identified are involved in critical platelet structures and functions, including cytoskeletal rearrangement and the coagulation cascade.

\section{Cytoskeletal proteins}

Previous reports have shown that tubulin B-1 chain is downregulated or overexpressed during the activation process, depending on the agonist used [15], and tubulin $\alpha 4 \mathrm{~A}$ chain is overexpressed in the secretome of activated platelets [16]. However, we found both of these tubulin chains were downregulated in platelets from patients with HTN compared to platelets from NTI. These observations suggest the platelets may be unable to maintain a discoid shapeeven under resting conditions-which may help to explain 


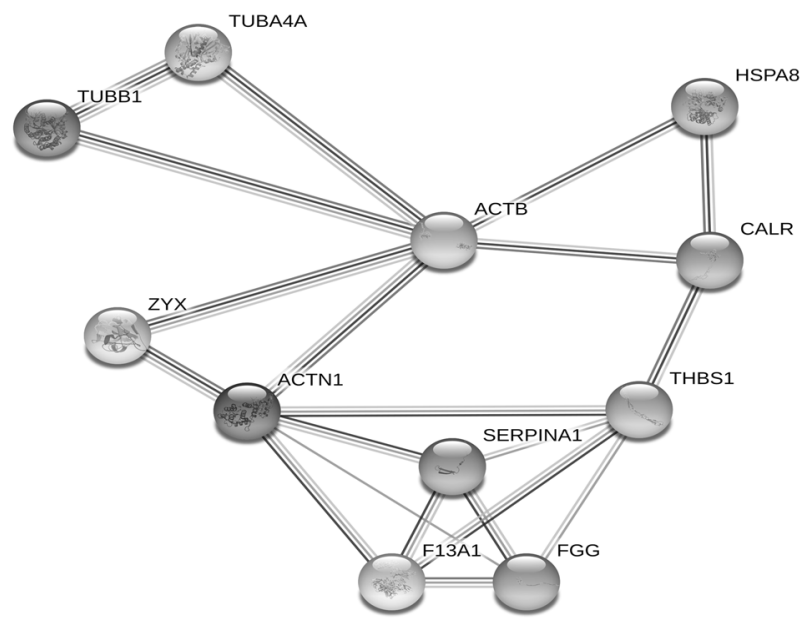

Fig. 5 Construction of the interactome. Network analysis of the 11 differentially expressed proteins (fold change $\geq 2$ ) in the platelets of patients with HTN compared to NTI using String software. Proteins are identified by their gene code. TUBB1 tubulin beta- 1 chain, TUBA4A tubulin $\alpha-4$ A chain, ZYX zyxin, ACTB cytoplasmic actin 1, HSPA8 cognate heat shock protein $71 \mathrm{kDa}$, ACTN1 alpha-actinin-1, CALR calreticulin, THBS1 thrombospondin-1, SERPINA1 alpha-1-antitrypsin, F13A1 coagulation factor XIII A chain, FGG fibrinogen gamma chain.

the morphological changes observed in the platelets of patients with HTN [7].

Previous proteomic analysis identified three spots that were downregulated in activated platelets compared to resting platelets, such as actin [15]. In contrast, we identified one overexpressed spot (HT11) as actin, and two downregulated spots (HT14 and HT15) were also identified as actin in platelets from patients with HTN.

Zyxin colocalizes and promotes the development of stress fibers and focal adhesions [17]. Actin and zyxin are part of the secretome of thrombin-activated platelets [18]. We detected low quantities of actin (HT14 and HT15) and zyxin (HT02) in the platelets of patients with HTN, which suggests that both proteins are released from the platelets of patients with HTN during their circulation in blood vessels, as previously reported for thrombin-activated platelets [16].

\section{Cytoskeleton-interacting proteins}

Calreticulin associates with actin, fibrinogen gamma chain, and coagulation factor XIII A chain. Calreticulin functions as a chaperone to regulate $\mathrm{Ca}^{2+}$ stores, and also binds to the $\mathrm{C}$-terminal cytoplasmic tail of $\alpha$-integrin to modulate its role in cell adhesion [19]. As in many biological systems, $\mathrm{Ca}^{2+}$ is a major messenger that promotes platelet activation, aggregation, and granule secretion [20]. The increased expression of calreticulin in the platelets of patients with HTN concurs with the previously described morphological changes and granule secretion [7]. In addition, upregulation of calreticulin could increase the risk of clots and thrombotic events in patients with HTN, as calreticulin stabilizes collagen-platelet interactions by associating with $\alpha 2 \beta 1$ and GPVI [21].

Fibrinogen chains participate in cytoskeletal reorganization by associating with filamin, vinculin, and alpha-actinin to promote clot retraction [22]. We found fibrinogen gamma chain was overexpressed and downregulated, in spots HT05 and HT08 respectively, in the platelets of patients with HTN compared to NTI. However, based on the registered LC-MS/MS scores, we decided to consider this protein is overexpressed (HT05). Overexpression of fibrinogen gamma chain in the platelets of patients with HTN may favor platelet aggregation by promoting activation and clustering of $\alpha \operatorname{Ilb} \beta 3$ and inducing thrombotic events.

The spots HT01 and HT02 were identified as coagulation factor XIII A chain (FXIII); HT01 was overexpressed in the platelets of patients with HTN. This result is in agreement with Majek et al. [15], who reported this protein was overexpressed in platelets activated in vitro with various agonists. FXIII translocates to the periphery of platelets, where it crosslinks and stabilizes the cytoskeleton of activated platelets [23]. We consider that overexpression of FXIII may promote the cytoskeletal reorganization and platelet shape changes observed in patients with HTN [7]. Collectively, these results suggest that overexpression of calreticulin, fibrinogen gamma chain, and FXIII promote integrin activation, platelet aggregation, and granule secretion-which favor thrombosis-in patients with HTN.

\section{Coagulation-system proteins}

Alpha-1-antitrypsin, fibrinogen gamma chain, and coagulation factor XIII A chain are related to the coagulation cascade and modulate the structural properties of clots to confer resistance to degradation [24, 25]. Our proteomic analysis showed that coagulation factor XIII A chain (HT01) and fibrinogen gamma chain (HT05) were overexpressed in platelets from patients with HTN, while fibrinogen gamma chain (HT08 and HT12) was downregulated. We identified three electrophoretic entities (HT05, HT08, and HT12) with different $\mathrm{pI}$ and MW values for fibrinogen gamma chain, while Májek et al. [15] identified eight different spots for this protein in activated platelets. The heterogeneity in the $\mathrm{pI}, \mathrm{MW}$, and function of this protein are likely the result of posttranslational modifications (proteolysis, phosphorylation, glycation, sulfation) [26]. Glycation of fibrinogen gamma chain increases the MW and resistance of this protein to fibrinolysis in patients suffering from type 2 diabetes mellitus. The differences observed for gamma fibrinogen spots in the platelets from patients with HTN may be due to glycation, which contributes to the formation of resistant, stable, rigid clots [27]. 
Alpha-1-antitrypsin is an anti-inflammatory protein and natural inhibitor of the coagulation system. Alpha-1antitrypsin activity can be inhibited by oxidation of its methionine residues [28]. Patients suffering high blood pressure generate high concentrations of reactive oxygen species (ROS), which creates oxidative stress [29]. Thus, high levels of ROS may inhibit alpha-1-antitrypsin and-in combination with reduced expression of alpha-1-antitrypsin in the platelets of patients with HTN-make thrombosis more likely to occur. In the present study, three differentially expressed spots suggest dysregulation of three isoforms of alpha-1-antitrypsin in the platelets of patients with HTN. Protein phosphorylation/dephosphorylation, the most commonly observed posttranslational modification in activated platelets, can alter the pI of proteins [13]. Alpha-1antitrypsin contains 15 phosphorylation sites and eight acetylation sites (phosphosite.com), thus posttranslational modifications may influence its MW.

We also identified a $71 \mathrm{kDa}$ heat shock protein cognate (Hsp70), $\alpha$-actinin-1, and thrombospondin-1. Although the changes in the expression of these proteins were not significant, alterations to these proteins could influence cytoskeletal organization and the coagulation cascade in the platelets of patients with HTN.

Hsp70 promotes protein folding and prevents protein aggregation [30]. Hsp70 translocates to the cytoskeleton in thrombin-activated platelets [22]. Although its role as a chaperon protein has not been described in platelets, Hsp70 promotes platelet activation, aggregation, and secretion [31]. We found Hsp70 (HT04) was overexpressed in platelets from patients with HTN, which could contribute to the constitutive platelet activation associated with these patients.

The actin-binding protein alpha-actinin acts as a scaffold for other proteins, such as vinculin, zyxin, and integrins, to form stress fibers. Corte et al. [16] reported that $\alpha$-actinin was overexpressed in platelets from patients with HTN. In contrast, we observed nonsignificant downregulation of alpha-actinin in platelets from patients with HTN, which may suggest a compensatory mechanism exists to diminish the risk of clot formation by constitutively activated platelets.

Thrombospondin-1 represents $25 \%$ of the proteins released from activated platelets. Overexpression of thrombospondin-1 has been reported in the secretome of thrombin-activated platelets in comparison to resting platelets [16]. Our LC-MS/MS analysis revealed nonsignificant downregulation of thrombospondin-1 (HT06) in platelets from HTN. Our results conflict with previous reports; however, we must consider that platelets are constitutively activated in patients with HTN and may constantly release thrombospondin-1 at low levels that are barely detectable. Released thrombospondin- 1 is trapped in the extracellular matrix (ECM) and overexpression of thrombospondin-1 is related to formation of atherosclerotic plaques. Enrichment of the ECM with thrombospondin-1 promotes cytoskeletal reorganization and extension of lamellipodia by myoblasts and endothelial cells; similarly, such events could promote platelets to form lamellipodia and filopodia [32].

To compensate for the small number of samples subjected to LC-MS/MS analysis, we selected an fc $\geq 2$. Nevertheless LC-MS/MS approach analyzes many proteins in a single experiment, this methodology is not suitable to assess large numbers of individuals.

Efforts to determine the complex changes in the platelet proteome during HTN include the induction of HTN in animal models; this strategy revealed modifications of the platelet proteome, but was unable to identify the specific effects on platelet proteome attributable to HTN [33].

High blood pressure values, the severity of the disease, and the frequency of treatment might have an impact on platelets protein expression. Patients included in our study were classified from controlled to grade 2 (Table 1), which could show less dramatic proteome changes. Our study provides a small insight into the biochemical events underlying platelets from HTN patients, and performing a proteomic study, considering not only the grade of HTN of the patient included and time of evolution of the disease but also the treatment they are receiving, would shed light on the accurate changes in platelets proteome induced by HTN. In addition, analysis of a larger sample number would provide more consistent results and help confirm the results of the present study. Overall, this study provides a better understanding of the pathophysiology of HTN and may help identify novel therapeutic targets.

\section{Conclusion}

This study, for the first time, assesses differential protein expression in resting platelets from patients with HTN and NTI. Our results demonstrate that deregulation of cytoskeletal and coagulation-system proteins affects the function of platelets and contributes to the pathophysiology of the disease. Further characterization of the effects of abnormal protein expression in platelets could potentially enable the development of novel therapeutic targets or biomarkers to control HTN.

\section{Summary}

\section{What is known about this topic}

- HTN is a complex and multifactorial disease with biophysical, biochemical, and morphological changes in blood cells. 
- HTN is characterized by activated platelets, which are related to cardiovascular morbidity and mortality.

\section{What this study adds}

- A differential protein expression of platelets from individuals with HTN compared to platelets from NTI.

- Platelets from individuals with HTN showed $273 \pm 9$ electrophoretic entities, in contrast to $163 \pm 9$ electrophoretic entities platelets from NTI.

- Differential protein expression involves cytoskeleton organization and coagulation cascade proteins.

Acknowledgements The authors would like to thank Alicia Chagoya, MSc, of CINVESATV, Irapuato, México, for conducing the LC-MS/ MS analysis. This work was supported by SIP, IPN, grant no. 20195020 .

\section{Compliance with ethical standards}

Conflict of interest The authors declare no competing interests.

Publisher's note Springer Nature remains neutral with regard to jurisdictional claims in published maps and institutional affiliations.

\section{References}

1. Tadic M, Cuspidi C, Mancia G, Dell'oro R, Grassi G. COVID-19, hypertension and cardiovascular diseases: should we change the therapy? Pharmacol Res. 2020;158:104906.

2. Poulter NR, Prabhakaran D, Caulfield M. Hypertension. Lancet. 2015;386:801-12.

3. Hong S, Adler KA, Von Kanel R, Nordberg J, Ziegler MG, Mills PJ. Prolonged platelet activation in individuals with elevated blood pressure in response to a moderate exercise challenge. Psychophysiology. 2009;46:276-84.

4. Jindra A Jr, Savlikova J, Bultas J. Relationship between plasma catecholamines and the renin-aldosterone system during exercise in normal and essential hypertensive subjects. Clin Exp Hypertens A. 1990;12:415-35.

5. White GC 2nd, Rompietti R. Platelet secretion: indiscriminately spewed forth or highly orchestrated? J Thromb Haemost. 2007;5: 2006-8.

6. Blann AD, Nadar S, Lip GY. Pharmacological modulation of platelet function in hypertension. Hypertension. 2003;42:1-7.

7. Cerecedo D, Martinez-Vieyra I, Sosa-Peinado A, Cornejo-Garrido J, Ordaz-Pichardo C, Benitez-Cardoza C. Alterations in plasma membrane promote overexpression and increase of sodium influx through epithelial sodium channel in hypertensive platelets. Biochim Biophys Acta. 2016;1858:1891-903.

8. Garcia-Rubio D, Rodriguez-Varela M, Martinez-Vieyra I, De La Mora MB, Mendez-Mendez JV, Duran-Alvarez JC, et al. Alterations to the contents of plasma membrane structural lipids are associated with structural changes and compartmentalization in platelets in hypertension. Exp Cell Res. 2019;385:111692.

9. Burkhart JM, Gambaryan S, Watson SP, Jurk K, Walter U, Sickmann A, et al. What can proteomics tell us about platelets? Circ Res. 2014;114:1204-19.
10. García A, Prabhakar S, Brock CJ, Pearce AC, Dwek RA, Watson SP, et al. Extensive analysis of the human platelet proteome by two-dimensional gel electrophoresis and mass spectrometry. Proteomics. 2004;4:656-68.

11. Varol E, Akcay S, Icli A, Yucel H, Ozkan E, Erdogan D, et al. Mean platelet volume in patients with prehypertension and hypertension. Clin Hemorheol Microcirc. 2010;45:67-72.

12. Bath PM, Butterworth RJ. Platelet size: measurement, physiology and vascular disease. Blood Coagul Fibrinolysis. 1996;7:157-61.

13. Sacristan D, Marques M, Zamorano-Leon JJ, Luque M, Armengol J, Del Castillo J, et al. Modifications by Olmesartan medoxomil treatment of the platelet protein profile of moderate hypertensive patients. Proteom Clin Appl. 2008;2:1300-12.

14. Rabilloud T, Lelong C. Two-dimensional gel electrophoresis in proteomics: a tutorial. J Proteom. 2011;74:1829-41.

15. Majek P, Reicheltova Z, Stikarova J, Suttnar J, Sobotkova A, Dyr JE. Proteome changes in platelets activated by arachidonic acid, collagen, and thrombin. Proteome Sci. 2010;8:56.

16. Corte AD, Maugeri N, Pampuch A, Cerletti C, De Gaetano G, Rotilio D. Application of 2-dimensional difference gel electrophoresis (2D-DIGE) to the study of thrombin-activated human platelet secretome. Platelets. 2008;19:43-50.

17. Hirata $H$, Tatsumi $H$, Sokabe M. Mechanical forces facilitate actin polymerization at focal adhesions in a zyxin-dependent manner. $\mathrm{J}$ Cell Sci. 2008;121:2795-804.

18. Coppinger JA, Cagney G, Toomey S, Kislinger T, Belton O, Mcredmond JP, et al. Characterization of the proteins released from activated platelets leads to localization of novel platelet proteins in human atherosclerotic lesions. Blood. 2004;103:2096-104.

19. Kwon MS, Park CS, Choi K, Ahnn J, Kim JI, Eom SH, et al. Calreticulin couples calcium release and calcium influx in integrin-mediated calcium signaling. Mol Biol Cell. 2000;11: 1433-43.

20. Stefanini L, Roden RC, Bergmeier W. CalDAG-GEFI is at the nexus of calcium-dependent platelet activation. Blood. 2009;114: 2506-14.

21. Elton CM, Smethurst PA, Eggleton P, Farndale RW. Physical and functional interaction between cell-surface calreticulin and the collagen receptors integrin alpha2beta1 and glycoprotein VI in human platelets. Thromb Haemost. 2002;88:648-54.

22. Gevaert K, Eggermont L, Demol H, Vandekerckhove J. A fast and convenient MALDI-MS based proteomic approach: identification of components scaffolded by the actin cytoskeleton of activated human thrombocytes. J Biotechnol. 2000;78:259-69.

23. Serrano K, Devine DV. Intracellular factor XIII crosslinks platelet cytoskeletal elements upon platelet activation. Thromb Haemost. 2002;88:315-20.

24. Undas A, Ariens RA. Fibrin clot structure and function: a role in the pathophysiology of arterial and venous thromboembolic diseases. Arterioscler Thromb Vasc Biol. 2011;31:e88-99.

25. Gaffney PJ, Whitaker AN. Fibrin crosslinks and lysis rates. Thromb Res. 1979;14:85-94.

26. Farley AR, Link AJ. Identification and quantification of protein posttranslational modifications. Methods Enzymol. 2009;463: 725-63.

27. Dunn EJ, Philippou H, Ariens RA, Grant PJ. Molecular mechanisms involved in the resistance of fibrin to clot lysis by plasmin in subjects with type 2 diabetes mellitus. Diabetologia. 2006;49:1071-80.

28. Matheson NR, Wong PS, Travis J. Enzymatic inactivation of human alpha-1-proteinase inhibitor by neutrophil myeloperoxidase. Biochem Biophys Res Commun. 1979;88:402-9.

29. El Haouari M, Rosado JA. Platelet function in hypertension. Blood Cells Mol Dis. 2009;42:38-43. 
30. Mayer MP, Bukau B. Hsp70 chaperones: cellular functions and molecular mechanism. Cell Mol Life Sci. 2005;62:670-84.

31. Rigg RA, Healy LD, Nowak MS, Mallet J, Thierheimer ML, Pang $\mathrm{J}$, et al. Heat shock protein 70 regulates platelet integrin activation, granule secretion and aggregation. Am J Physiol Cell Physiol. 2016;310:C568-75.
32. Riessen R, Kearney M, Lawler J, Isner JM. Immunolocalization of thrombospondin-1 in human atherosclerotic and restenotic arteries. Am Heart J. 1998;135:357-64.

33. Gebhard S, Steil L, Peters B, Gesell-Salazar M, Hammer E, Kuttler B, et al. Angiotensin II-dependent hypertension causes reversible changes in the platelet proteome. J Hypertens. 2011;29:2126-37. 